Review began 02/24/2022 Review ended 03/01/2022 Published 03/06/2022

๑) Copyright 2022

Masoudi et al. This is an open access article distributed under the terms of the Creative Commons Attribution License CCBY 4.0., which permits unrestricted use, distribution, and reproduction in any medium, provided the original author and source are credited.

\section{Point-of-Care Ultrasound for Diagnosis and Pain Control of Sternal Fracture}

\author{
Aidin Masoudi ${ }^{1}$, Leily Naraghi $^{1}$ \\ 1. Emergency Medicine, Maimonides Medical Center, Brooklyn, USA
}

Corresponding author: Aidin Masoudi, aidin.masoudi@gmail.com

\section{Abstract}

In this case report, point-of-care ultrasound (POCUS) was performed to diagnose a sternal fracture and to perform an ultrasound-guided hematoma block on an elderly patient through which excellent pain control was achieved. POCUS is a valuable tool in expediting the diagnosis of sternal fracture and can be used to safely perform a hematoma block for pain control.

Categories: Emergency Medicine, Pain Management, Trauma

Keywords: pain control, trauma, hematoma block, sternal fracture, point-of-care ultrasound

\section{Introduction}

A sternal fracture occurs due to trauma to the chest wall and can be isolated or in conjunction with other injuries [1,2]. It is reported in $8 \%-10 \%$ of patients suffering from blunt chest wall trauma [1-4]. These injuries can be severely painful and lead to shallow breathing, especially in elderly patients, and commonly results in the use of narcotics for pain control [2]. The reduced vital capacity and the use of narcotics can increase the risk of complications such as pneumonia and falls $[5,6]$. This is especially true in the elderly populations where mortality related to chest trauma, including pulmonary complications, is about $38 \%$ [5-7].

POCUS can diagnose a sternal fracture accurately and expeditiously [2,3]. Studies show that it takes less than one minute to three minutes to perform and diagnose a sternal fracture at the bedside $[2,8,9]$. Opioid sparing analgesia and a multimodal pain control approach are key components in preventing complications related to chest wall trauma and sternal fractures $[6,10]$. Ultrasound-guided sternal hematoma block is a safe and quick intervention that can play a significant role in the multimodal pain control approach [2]. This can reduce morbidity, mortality, and hospital stay for elderly patients suffering from sternal fracture $[2,10]$.

This case report demonstrates the use of POCUS for diagnosis and pain control via hematoma block of a sternal fracture in an elderly patient.

\section{Case Presentation}

An 83-year-old female presented to the emergency department (ED) with chest wall pain after falling on a bus. She fell from standing and hit her chest on the edge of a seat. On exam, she had significant tenderness over her sternum. Her chest X-ray was unremarkable.

Due to a strong clinical suspicion for a sternal fracture, a POCUS was performed in the ED (Video 1), revealing a sternal fracture (Figure 1).

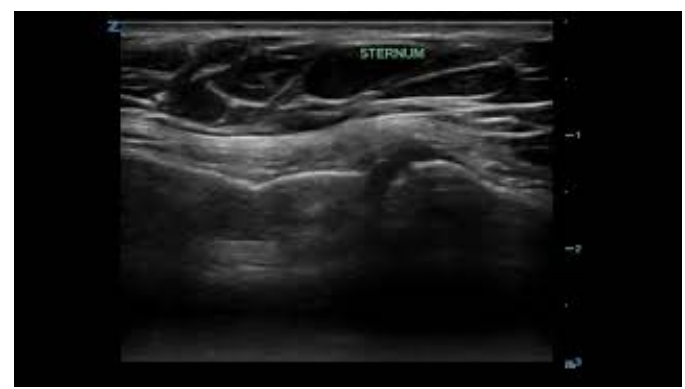

VIDEO 1: Ultrasound clip of ternal fracture.

View video here: https://youtu.be/eLoU2JIVcag 


\section{Cureus}

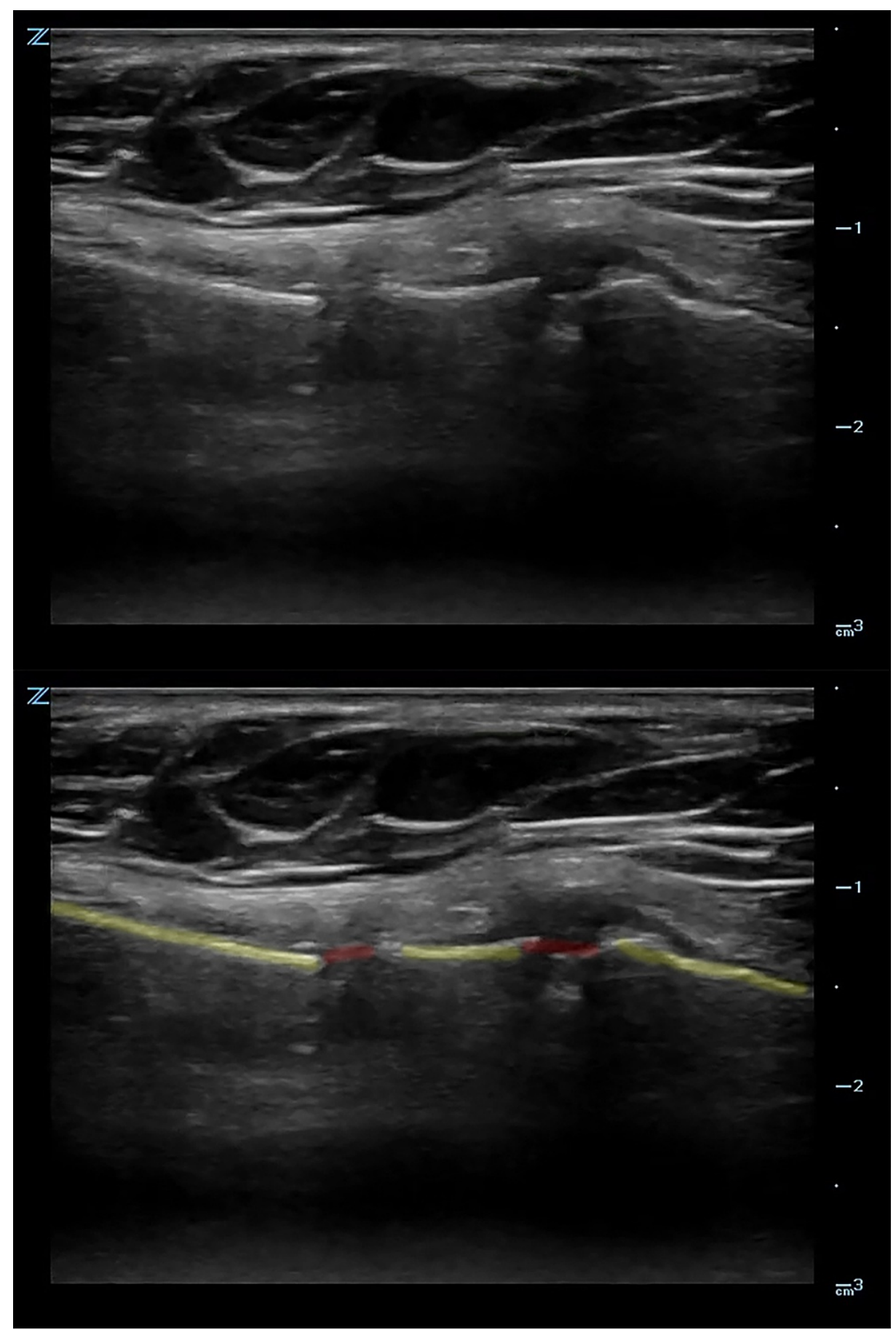

FIGURE 1: Ultrasound image of two areas of cortical disruption visualized on the sternum with overlying hematoma. The anterior cortex of the sternum (yellow) and the fracture sites (red).

The sternal fracture was confirmed by chest computed tomography (CT) (Figure 2). 


\section{Cureus}

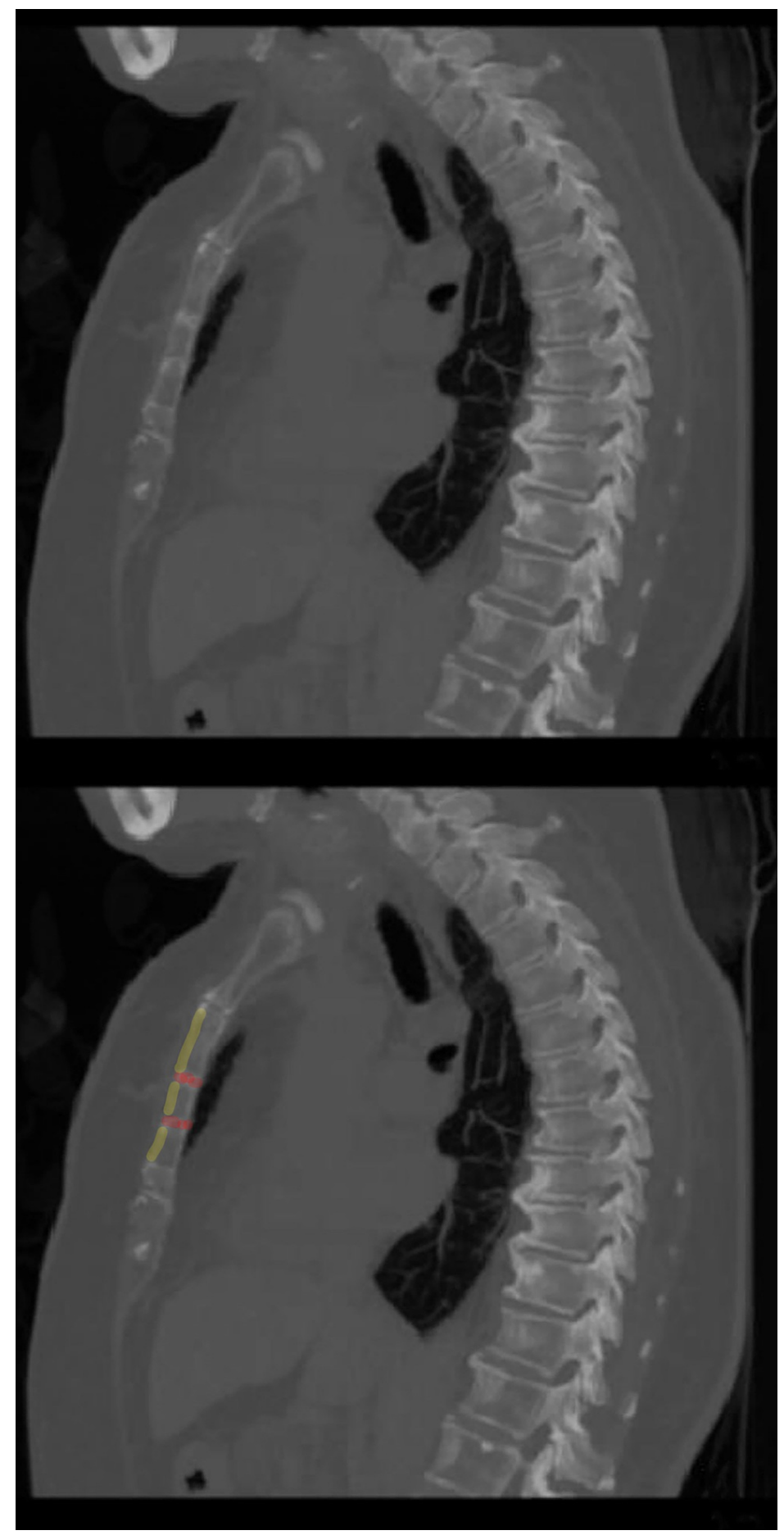

FIGURE 2: Sagittal view of the chest computed tomography. The anterior cortex of the sternum (yellow) and the fracture sites (red) are highlighted.

An ultrasound-guided sternal hematoma block was performed (Figure 3), and 10cc of bupivacaine $0.5 \%$ was injected at the site of hematoma overlying the fracture (Figure 4, Video 2). 


\section{Cureus}

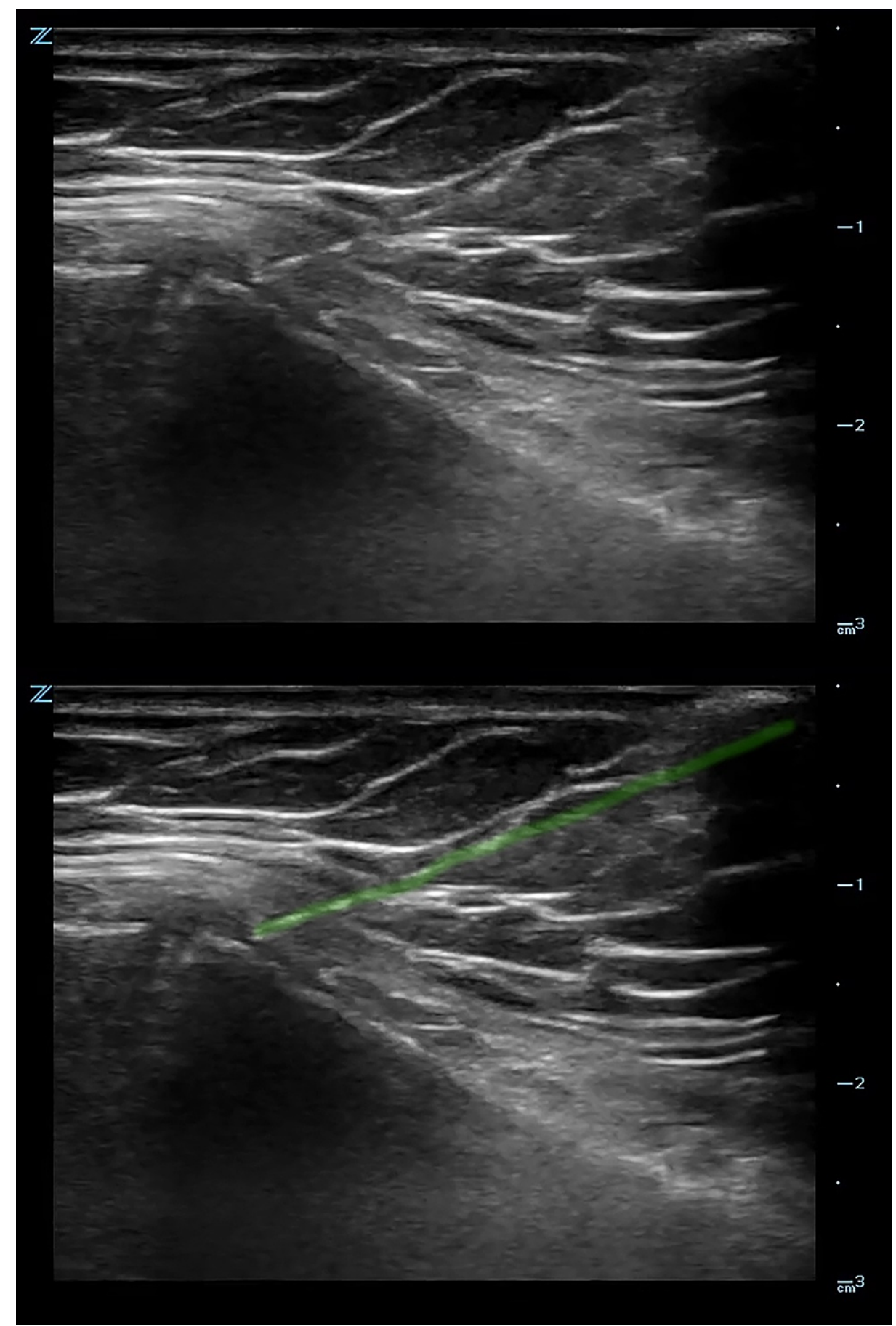

FIGURE 3: Ultrasound image of in-line needle insertion in the hematoma overlying the fracture. The needle is highlighted (green). 


\section{Cureus}

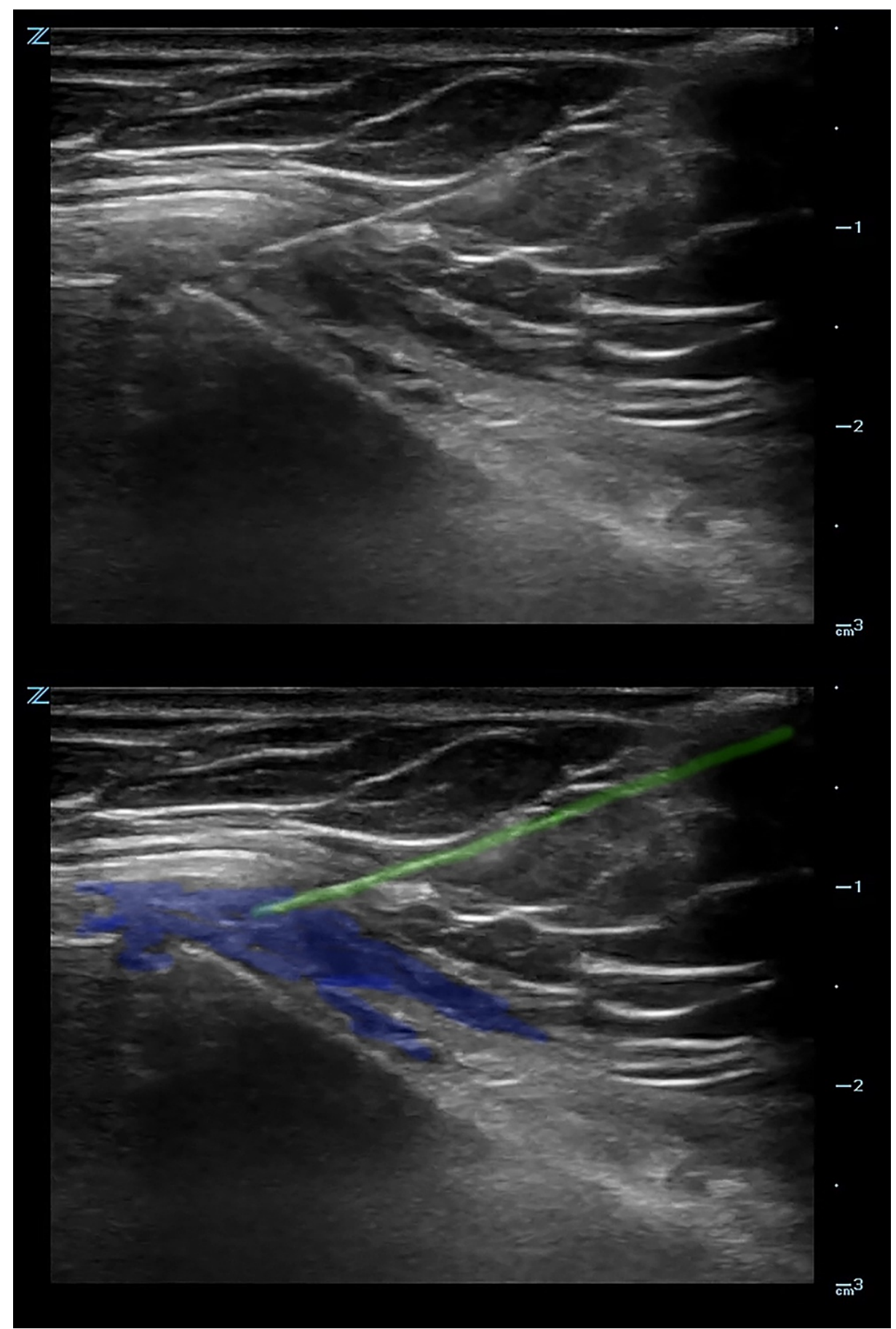

FIGURE 4: Ultrasound image after injection of local anesthetic into the hematoma. The needle (green) and the injected anesthetic (blue) are highlighted.

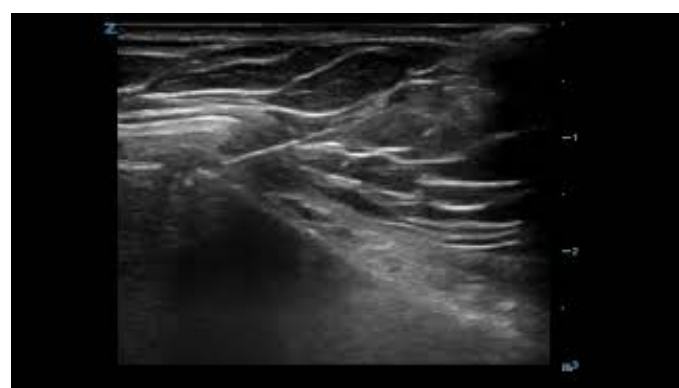


VIDEO 2: Ultrasound clip showing the needle being directed in-plane to the fracture site.

View video here: https://youtu.be/jlqHR26dal8

Immediately after the procedure, the patient reported complete resolution of her pain and was admitted to the hospital for 24-hour observation after which she was safely discharged home.

\section{Discussion}

POCUS is an important tool not in the diagnosis and treatment of a variety of pathologies in the field of emergency medicine (EM). The expedited nature of POCUS, in addition to having no ionizing radiation, is extending its reach beyond EM practice. In addition, POCUS is often more accurate than X-rays for diagnosing sternal fractures, rib fractures, clavicular fractures, pneumothorax, hemothorax, and pleural effusions $[2,8,9,11-15]$.

The flat, broad, and plane-like structure of the sternum combined with the minimal overlying soft tissue make the sternum anatomically ideal for the ultrasound diagnosis of fracture and for performing the ultrasound-guided hematoma block $[2,8,9]$. The limitation of ultrasound in the diagnosis of sternal fractures is the inability to estimate the severity of the injury and to rule out fracture-dislocation $[2,9]$. The severity of the sternal fracture can be estimated by the displacement of the fracture, which cannot be accurately measured by ultrasound when the displacement exceeds the thickness of the sternum [2,9]. This limitation makes chest CT necessary when sternal fracture-dislocation or other coexisting injuries are suspected [2].

The multimodal pain control approach is becoming a gold standard in pain management, especially in the elderly population [16]. Regional anesthesia, including ultrasound-guided hematoma block, can significantly reduce the need for opioid use in patients suffering from sternal fractures, which can reduce their mortality, morbidity, and length of hospital stay $[2,6,7,10]$.

\section{Conclusions}

POCUS is a valuable tool in expediting the diagnosis of sternal fractures and can be utilized to safely perform hematoma blocks for pain control.

\section{Additional Information \\ Disclosures}

Human subjects: Consent was obtained or waived by all participants in this study. Conflicts of interest: In compliance with the ICMJE uniform disclosure form, all authors declare the following: Payment/services info: All authors have declared that no financial support was received from any organization for the submitted work. Financial relationships: All authors have declared that they have no financial relationships at present or within the previous three years with any organizations that might have an interest in the submitted work. Other relationships: All authors have declared that there are no other relationships or activities that could appear to have influenced the submitted work.

\section{References}

1. Trinca GW, Dooley BJ: The effects of mandatory seat belt wearing on the mortality and pattern of injury of car occupants involved in motor vehicle crashes in Victoria. Med J Aust. 1975, 1:675-678. 10.5694/j.13265377.1975.tb111734.x

2. Wilson SR, Price DD, Penner E: Pain control for sternal fracture using an ultrasound-guided hematoma block. J Emerg Med. 2010, 38:359-61. 10.1016/j.jemermed.2008.03.006

3. Engin G, Yekeler E, Güloğlu R, Acunaș B, Acunaș G: US versus conventional radiography in the diagnosis of sternal fractures. Acta Radiol. 2000, 41:296-9. 10.1080/028418500127345280

4. Jackson M, Walker WS: Isolated sternal fracture: a benign injury? . Injury. 1992, 23:535-536. 10.1016/00201383(92)90154-k

5. Khoriati AA, Rajakulasingam R, Shah R: Sternal fractures and their management . J Emerg Trauma Shock. 2013, 6:113-6. 10.4103/0974-2700.110763

6. Davis K, Riley B, Connor X, Mitchell A, Abi-Fares C, Basson W, White L: Subpectorial parasternal plane block catheters for the management of sternal fractures in the context of chest trauma: a case series. J Clin Anesth. 2021, 71:110236. 10.1016/j.jclinane.2021.110236

7. Witt CE, Bulger EM: Comprehensive approach to the management of the patient with multiple rib fractures: a review and introduction of a bundled rib fracture management protocol. Trauma Surg Acute Care Open. 2017, 2:e000064. 10.1136/tsaco-2016-000064

8. Fenkl R, von Garrel T, Knaepler H: Emergency diagnosis of sternum fracture with ultrasound . Der Unfall. 1992, 95:375-379.

9. Hendrich C, Finkewitz U, Berner W: Diagnostic value of ultrasonography and conventional radiography for the assessment of sternal fractures. Injury. 1995, 26:601-604. 10.1016/0020-1383(95)00110-u

10. Coary R, Skerritt C, Carey A, Rudd S, Shipway D: New horizons in rib fracture management in the older 


\section{Cureus}

adult. Age Ageing. 2020, 49:161-7. 10.1093/ageing/afz157

11. Bitschnau R, Gehmacher O, Kopf A, Scheier M, Mathis G: Ultrasound diagnosis of rib and sternal fractures . Ultraschall Med. 1997, 18:158-61. 10.1055/s-2007-1000416

12. Jin W, Yang DM, Kim HC, Ryu KN: Diagnostic values of sonography for assessment of sternal fractures compared with conventional radiography and bone scans. J Ultrasound Med. 2006, 25:1263-8. 10.7863/jum.2006.25.10.1263

13. Mahlfeld A, Franke J, Mahlfeld K: Diagnosis of sternal fractures by ultrasound . Zentralbl Chir. 2001, 126:624. 10.1055/s-2001-11727

14. Sferopoulos NK: Fracture separation of the medial clavicular epiphysis: ultrasonography findings . Arch Orthop Trauma Surg. 2003, 123:367-9. 10.1007/s00402-003-0542-8

15. Wüstner A, Gehmacher O, Hämmerle S, Schenkenbach C, Häfele H, Mathis G: Ultrasound diagnosis in blunt thoracic trauma. Ultraschall Med. 2005, 26:285-90. 10.1055/s-2005-858524

16. Santini A, Petruzzo A, Giannetta N, Ruggiero A, Di Muzio M, Latina R: Management of chronic musculoskeletal pain in veterans: a systematic review. Acta Biomed. 2021, 92:e2021011.

10.23750/abm.v92iS2.11352 